\title{
Current Developments in the Therapy of Protozoan Infections
}

\author{
Mario Zucca and Dianella Savoia*
}

Department of Clinical and Biological Sciences, University of Torino, Italy

\begin{abstract}
Protozoan parasites cause serious human and zoonotic infections, including life-threatening diseases such as malaria, African and American trypanosomiasis, and leishmaniasis. These diseases are no more common in the developed world, but together they still threaten about $40 \%$ of the world population (WHO estimates). Mortality and morbidity are high in developing countries, and the lack of vaccines makes chemotherapy the only suitable option. However, available antiparasitic drugs are hampered by more or less marked toxic side effects and by the emergence of drug resistance. As the main prevalence of parasitic diseases occurs in the poorest areas of the world, the interest of the pharmaceutical companies in the development of new drugs has been traditionally scarce. The establishment of public-private partnerships focused on tropical diseases is changing this situation, allowing the exploitation of the technological advances that took place during the past decade related to genomics, proteomics, and in silico drug discovery approaches. These techniques allowed the identification of new molecular targets that in some cases are shared by different parasites. In this review we outline the recent developments in the fields of protease and topoisomerase inhibitors, antimicrobial and cell-penetrating peptides, and RNA interference. We also report on the rapidly developing field of new vectors (micro and nano particles, mesoporous materials) that in some cases can cross host or parasite natural barriers and, by selectively delivering new or already in use drugs to the target site, minimize dosage and side effects.
\end{abstract}

Keywords: Protozoa, protease, topoisomerase, RNAi, nanovectors.

\section{INTRODUCTION}

Protozoan parasitic diseases remain an unsolved public health problem, especially in tropical regions. The major death toll is due to malaria, leishmaniasis, and African and American trypanosomiasis, whose high mortality rates in developing countries are associated to poor sanitary conditions and lack of efficient prophylactic measures [1]. In the so-called developed countries these diseases were since long eradicated, but some of them are reappearing as opportunistic infections in immunodeficient patients or as emerging diseases related to tourist exchange, immigration flows from endemic areas and climate changes linked to global warming [2]. Despite the remarkable amount of research aimed at the production of protective vaccines, the success is still elusive and chemotherapy remains the mainstay for the treatment of these diseases. However, many of the drugs currently in use were introduced decades ago, and most of them suffer of serious disadvantages. Because the main prevalence of parasitic diseases occurs in the poorest areas of the world, the market has not enough strength to drive the development of new drugs. In 2000, only about $0.1 \%$ of investment in health research was related to malaria, leishmaniasis, trypanosomiasis and tuberculosis, whereas the cumulative contribution of these diseases to the global disease burden is 5\% [1].

\footnotetext{
*Address correspondence to this author at the Department of Clinical and Biological Sciences, Faculty of Medicine San Luigi Gonzaga, University of Torino at S. Luigi Gonzaga Hospital, 10043 Orbassano (TO), Italy; Tel: +39-0116705427; Fax: +39-0119038639;

E-mail: dianella.savoia@unito.it
}

Notwithstanding these handicaps, in recent years the search for antiparasitic drugs received new impulse thanks to new technical and political developments. Among these, particularly important have been the publicly-founded sequencing of parasitic genomes and the establishment of public-private partnerships focused on tropical diseases [1].

The studies on host-parasite interactions at the cellular level have been revolutionized by the use of reporter genes, from $\beta$-gal to luciferase and fluorescent proteins [3]. This technology provided new impetus to the development of reliable drug screens, and a WHO program for training in tropical diseases research has initiated a network based on reporter gene technology to hasten the process of drug discovery [3]. The availability of the fully sequenced and assembled genomes of Plasmodium, Leishmania and Trypanosoma species provides a multidisciplinary approach based on structure-function studies of proteins and high-through-put screening of drug libraries. In theory, the possibility to compare parasitic genomes and the in deep knowledge of parasite metabolism allow the development of new drugs that target biochemical processes common to different parasites. This strategy, when possible, would produce huge savings in the development, toxicity testing and marketing procedures. Accordingly, many recently established lines of research are primarily focused on the identification of new targets and on the subsequent screening of thousands of substances to find active molecules suitable for human therapy.

In this short review we will report on protease and topoisomerase inhibitors, antimicrobial peptides, and on new ap- 
proaches based on RNA interference, proteomics and nanodrug delivery.

\section{PROTEASE INHIBITORS}

Proteases, a large group of ubiquitous enzymes involved in a multitude of physiological reactions, ranging from protein catabolism to activation or signal functions, have recently been identified as druggable targets. Many protease inhibitors are currently used in antiretroviral therapy and more are being developed to treat hypertension, diabetes, thrombosis, osteoporosis, infectious diseases, and cancer. Several proteases have also been identified as promising targets by genetic or chemical knock-out studies in protozoan parasites [4]. As homologous proteases can perform similar functions in different parasites, it can be envisaged that in some cases a single inhibitor could be used for more than one disease among malaria, trypanosomiasis, leishmaniasis, toxoplasmosis, cryptosporidiosis, and amebiasis. Protozoan proteases are involved in the cell metabolism or in the interaction with the host, behaving in this case as true virulence factors. On this assumptions, the selective inhibition of crucial protozoan proteases is one of the most promising strategies to develop new therapies against Plasmodium, Leishmania and Trypanosoma.

Essential processes unique to the Plasmodium and not to the host represent good targets for new antimalarial drugs. The sequencing of the Plasmodium genome brought into light many new potential targets, that can be broadly classified into three categories: targets involved in membrane transport and signalling, targets involved in metabolic processes, and targets involved in protein degradation [5]. Malarial proteases can be divided into two functional groups: proteases involved in erythrocyte invasion and rupture, and proteases involved in haemoglobin degradation [6]. Cysteine protease $(\mathrm{CP})$ inhibitors such as the epoxysuccinyl peptide E64 from Aspergillus japonicum, or leupeptin (an actinomycete-derived tetrapeptide) and chymostatin (a streptomycetederived mixture of three oligopeptides) block erythrocyte rupture, causing the clustering of intracellular merozoites. These molecules can be used as templates for the development of new inhibitors specific to individual plasmodial proteases [6]. One of the best studied plasmodial metabolic pathways is the process of haemoglobin degradation [7]. Within the intra-erythrocytic form of the parasite the haemoglobin is degraded by papain-like CPs (falcipains) and aspartic proteases (plasmepsins) to amino acids utilized by the parasite to grow and replicate [7]. The P. falciparum falcipains involved in haemoglobin catabolism (falcipain-2, falcipain-2', and falcipain 3) are recognized as promising targets of new antimalarial drugs [8]. Some compounds of the azadipeptide nitrile family display in vitro structuredependent antimalarial activity against both chloroquinesensitive and chloroquine resistant $P$. falciparum by inhibiting falcipain 2 and 3 [7]. Malaria plasmepsins are responsible for the initial cleavage of haemoglobin within the food vacuole of the parasite during its erythrocytic stage. In $P$. falciparum there are ten different plasmepsin genes, that share a $65-70 \%$ sequence homology [9]. Due to this gene redundancy, the so far discovered single plasmepsin inhibitors are not able to completely stop parasite growth, and this is the main reason for the delay of their development as drugs. To achieve parasite starvation, the ideal inhibitor should be simultaneously active against plasmepsins I, II, IV and the structurally related histo-aspartic protease HAP, but inactive against the most closely related human aspartic proteases (cathepsins D and E), that share 35\% sequence homology with parasite plasmepsins [5]. Starting from the three-dimensional structure of $P$. falciparum plasmepsin II, a series of compounds of the bicyclic diamine family, active against the above mentioned $P$. falciparum plasmepsins but inactive on human cathepsins, have been developed [10]. The screening and testing of new plasmepsins inhibitors is performed by many research teams using the most up-to date techniques, such as proteomics [11] or the computational in silico approach $[5,12,13]$. By using the proteomics approach, Liu et al. have recently identified a $P$. falciparum plasmepsin inhibitor, G16, with an $\mathrm{IC}_{50}$ value of $937.5 \mathrm{nM}$ and no apparent cytotoxicity against common mammalian cell lines [11]. The inhibitory effect of this compound has been tested in vitro on human parasite-infected RBC. Results showed a dose-dependent, marked decrease in the number of newly formed ring-stage parasites, and at the same time an increase of free extracellular merozoites. These results need to be fully investigated prior to further development of the compound.

Proteases also play multiple roles in the pathogenesis of Chagas disease. The complete sequentiation of $T$. cruzi genome showed the presence of seventy CPs, forty serine peptidases, about two hundred and fifty metallopeptidases (most of them presenting homology to leishmanolysin), twenty five threonine peptidases and two aspartic peptidases [14]. Among these, cruzipain, a lysosomal enzyme belonging to type I CPs, is the best characterized and is currently considered a relevant candidate as a drug target and as a vaccine component [14]. The agents of African trypanosomiases share a major CP termed rhodesain in $T$. $b$. rhodesiense and brucipain in $T$. b. brucei, both belonging to the cathepsin $\mathrm{L}$ subfamily of the papain-like CPs and showing a $98.4 \%$ homology in protein sequence [15]. Effective inhibitors include thiosemicarbazones, that have a reversible effect, and peptidyl-vinyl sulfones, peptidyl-epoxychetones, and peptidylepoxysuccinates that irreversibly inactivate the enzyme active site [15]. The recently investigated aziridinyl peptides belonging to the family of peptidomimetic $\mathrm{CP}$ irreversible inhibitors are active against the above mentioned trypanosome major CPs [15], plasmodial falcipain 2 [16] and leishmania CPs [17]. Some of these compounds are highly selective for cathepsin-like parasite CPs and have been shown to impair the in vitro growth of $L$. major promastigotes and to decrease the infection rate of macrophages. Moreover, these compounds modulate cytokine secretion and stimulate NO production by infected macrophages [17].

\section{TOPOISOMERASE INHIBITORS}

DNA topoisomerases are ubiquitous enzymes involved in DNA replication, transcription, recombination and repair processes. They can be divided into two categories: DNA topoisomerases type I and type II. Eukaryotic cell type I DNA topoisomerases are monomeric ATP-independent enzymes active on positively and negatively supercoiled DNA by introducing single-stranded breaks followed by passage and rejoining. They can be divided into type IA, that cova- 
lently bind to the 5' end of the broken DNA strand, and type IB that bind to the 3' end. Leishmania and Trypanosoma type IB topoisomerases have a peculiar heterodimeric bisubunit structure, unique in nature, which makes them very attractive as drug targets $[2,18]$. Type II topoisomerases are ATP-dependent enzymes that introduce transient double strand breaks in the double helix followed by passage and rejoining. They can also be divided into type II $\alpha$, with homodimeric structure, and type II $\beta$, with heterotetrameric structure [2].

Topoisomerase inhibitors fall into two general categories: compounds that stimulate the formation of covalent enzymeDNA complexes (topoisomerase poisons or class I inhibitors), and compounds that interfere with the enzymatic functions (class II inhibitors). The identification of topoisomerases as promising drug targets is based on the clinical success of camptothecin derivatives as anticancer agents. Camptothecin, a natural pentacyclic alkaloid obtained from the barks and stem of the Chinese plant Camptotheca accuminata, exerts a strong inhibition on a wide range of type I topoisomerases. The molecule as such has heavy toxic sideeffects, but it is the progenitor of a large family of antitumour derivatives, some of which are currently used in anticancer therapy. Camptothecin is a potent poison of $P$. falciparum and T. brucei topoisomerase IB. Two of its derivatives, irinotecan and topotecan, which are in use as antitumour drugs, showed a reduced cytotoxic effect against $T$. bruce $i$ trypomastigotes, probably due to low intake into the parasite [19]. Camptothecin has been shown to inhibit the growth of L. donovani promastigotes in vitro [20], and studies performed in a mouse model of visceral leishmaniasis showed that the i.p. administration of liposome-encapsulated camptothecin at a dose of $2.5 \mathrm{mg} / \mathrm{kg}$ body weight induced a significant reduction of the parasitic burden in spleen and liver [21]. Sen et al. elucidated camptothecin mechanism of action in L. donovani, showing that by inhibiting type IB topoisomerase it starts a cascade of events resulting in cellular apoptosis [22]. Some topoisomerase II inhibitors such as ofloxacin, m-amsacrine and DW6, a DNA quadruplex binder, are also active against Trichomonas vaginalis, whose resistance against currently used drugs is increasing worldwide [23].

\section{ANTIMICROBIAL PEPTIDES}

Antimicrobial peptides (AMPs) are evolutionary conserved factors [24] that participate to the innate immune response representing the first line of defence against pathogens, and combine antimicrobial activity with angiogenic, immunomodulating and anti-inflammatory properties [25]. The concept of using AMPs as therapeutic tools was first introduced in the late $1990 \mathrm{~s}$, however none of them has yet reached the market [26]. Following the discovery, some 20 years ago, that magainins and cecropins display antiparasitic activities, many studies suggest that AMPs represent a promising starting point to develop new and efficient antiparasitic drugs [27]. Most known AMPs have been found in the skin of amphibians, but they are also produced by skin and mucosal surfaces of mammals in response to infections. The AMP exact mechanism of action has yet to be established, but, according to the most accepted model, the initial phase consists in an electrostatic interaction with the target cell, followed by massive accumulation and insertion into the plasma membrane. Subsequently, cecropins and magainins kill microorganisms by membrane permeabilization, with a detergent-like effect accompanied by pore formation [28-30]. This mechanism is quite rapid, concentration-dependent and, most important, it does not need to interact with a specific receptor, thus avoiding the selection of resistant strains. The AMP specificity of action relies on the differences in the composition and physicochemical properties of germ and host cell membranes. The possibility to select resistant proteinase-producer strains is unlikely, due to the fast kinetics of the process. Protozoan resistance mechanisms could more probably be of the barrier type, including the ability to form cysts (Entamoeba, Giardia, Toxoplasma, Cryptosporidium), the production of glycocalix (Trypanosomatidae), and the suitability to intracellular parasitism (Plasmodium, Leishmania, T. cruzi, Toxoplasma, Cryptosporidium). Many AMPs of different origin have been shown to be active against protozoa: Plasmodium falciparum is sensitive to magainin 2 and dermaseptin S3 and S4; T. cruzi to phylloseptin O1 and $\mathrm{O} 2$ and to dermaseptin D11 and D12; C. parvum to magainin 2, buforin 2 , and ranalexin $1 \mathrm{CB} ;$ L. mexicana to dermaseptin $\mathrm{S} 1$; L. amazonensis to dermaseptin $\mathrm{O} 1$ and $\mathrm{H} 3 ;$ L. major to skin peptide YY and to dermaseptin S1, S4 and to their synthetic analogues; $L$. infantum to temporin $1 \mathrm{Sa} ;$ L.donovani to bombinin $\mathrm{H} 2$ and $\mathrm{H} 4$, and to temporin $\mathrm{A}$ and $\mathrm{B}[31,32]$.

A second mechanism of action of AMPs involves the recognition of intracellular targets. These peptides cross the plasma membrane without permanently harming it, and recognize intracellular targets such as DNA (buforin), DnaK chaperone (drosocin and phyrrhocorrycin), and mitochondria (histatin 5) [33]. Histatin 5 is a human salivary AMP that targets fungal mitochondria and is also active against Leishmania, in which it induces a decrease of mitochondrial ATP synthesis ending in cell death [33]. This molecule is considered very interesting because it behaves as a true cellpenetrating peptide (a class of peptides more extensively treated below) that can be exploited to ferry a wide variety of cargo molecules (peptides, proteins, nucleic acids, and even nanoparticles) across the cell membrane [33]. Among AMP advantages we can list their rapid action against a broad spectrum of pathogens and their low tendency for resistance development, but their practical use is limited by serious drawbacks concerning difficult synthesis, metabolic stability, immunogenicity, bioavailability, route of administration, and production cost [34]. Most of these disadvantages are related to AMP large size, so the research in this field is currently devoted to design and develop smaller synthetic peptidomimetics [34-36].

\section{NEW APPROACHES}

Whereas traditional methods to generate genetic knockouts in protozoa are time-consuming, RNA interference (RNAi) can easily inhibit the expression of a target gene even if it is present in multiple copies, and as such is a simpler and faster method to achieve genetic turn off. The exploitation of this technique allowed the discovery of hundreds of host genes not previously known to be involved in host-pathogen interactions [37]. By now RNAi is extensively used to probe for protein functions [38, 39], to validate new drug targets [40] and to search for novel cell cycle regulators 
in $T$. brucei [41]. RNAi has been also used to elucidate the mechanisms that allow $L$. donovani survival inside phagocytic cells [42], and to investigate the genetic base of resistance to malaria parasites in different anopheles strains [43].

Beyond its function as a valuable tool for gene function analysis, it can be envisaged that RNAi could be exploited for therapeutic purposes by directly inhibiting parasite gene expression with small interfering iRNAs (siRNAs) used as drugs. The introduction of a specific double stranded RNA into $T$. brucei by electroporation triggered a transient specific silencing of a cognate gene [44]. By the use of an integratable vector containing a tetracycline-inducible promoter and a DNA sequence producing dsRNA as a stem-loop structure, Wang et al. obtained a stably maintained inhibition of $T$. brucei gene expression [45], whereas Claser et al. by RNAi-mediated silencing of cytoketarin 18 gene inhibited $T$. cruzi intracellular replication [46].

The major limitation to the clinical application of RNAi is the poor permeability of cell membranes to negatively charged nucleic acids. To circumvent this problem, research has focused on the development of efficient delivery strategies, of which one of the most promising seems the nanovector-based technique. Recently Aouadi M et al. demonstrated that in mice it is possible to target macrophages by orally delivering siRNAs encapsulated in glucan nanoparticles, obtaining a significant suppression of LPS-induced TNF- $\alpha$ production [47]. A second siRNA delivery technique exploits the carrier capacities of cell-penetrating peptides (CPPs) [48]. The existence of CPPs, also referred to as protein transduction domains, was inferred from the observation that the HIV-1 Tat-protein was able to cross the plasma membrane by itself $[49,50]$. Thereafter, a wide variety of CPPs derived from natural proteins have been discovered, and many others have been designed [51]. So far, CPPs can be grouped into two classes, of which the first requires covalent linkage with the transported drug and has been validated for protein and peptide delivery, whereas the second, involving the formation of stable but non covalent linkages with the drug, allowed the introduction of oligonucleotides and siRNAs on preclinical states [52]. The CPP-based strategy has several advantages, including rapid and highly efficient intracellular delivery, stability of the complex in serum and physiological buffers, and lack of toxicity. Beyond the common CPP ability to deliver intracellular drugs with no membrane damage, some peptides such as pVEC and TP10 possess direct antimicrobial activity against Candida albicans, Staphylococcus aureus and Mycobacterium smegmatis [53]. More recently Arrighi et al. demonstrated that TP10 is active against the blood and mosquito stages of $P$. falciparum and against the blood stage of T. brucei brucei [54].

The trypanosomatid genome sequentiation boosted the use of proteome-based techniques, that markedly contribute to our understanding of parasite biology, host-parasite interactions, and drug action mechanisms. For most antimalarial drugs, neither mode of action nor mechanisms of resistance development are fully elucidated, although these would be important prerequisites for successfully developing urgently required novel antimalarials [55]. The mechanisms underlying $P$. falciparum drug resistance have been investigated by analyzing the protein expression changes in trophozoites following treatment with cloroquine and artemisine [55]. These experiments were set up to develop a large-scale quantitative proteomic approach to study cellular responses to drug pressure, and allowed the preliminary identification of two sets of proteins up- and down-regulated in treated parasites.

The $T$. brucei dominant factor responsible for resistance to the trypanolytic activity of human serum has been identified as a protein named SRA for Serum ResistanceAssociated [56]. This protein is a truncated version of the VSG (the parasite variant surface glycoprotein) and is located in the endosomes, where it neutralizes human apolipoprotein L1, a factor that induces parasite death by osmotic effect. Experiments performed in mouse models of T. brucei infection have shown that the injection of a conjugate combining the pore-forming domain of apoL1 with the antigenbinding region of a specific camelid antibody can block the course of infection [57]. Proteomic screen techniques are also very useful to identify proteins responsible for resistance to in-use drugs. For example, El Fadili et al. observed that the expression of argininosuccinate synthetase (ARGG) was increased in a drug-resistant strain of $L$. infantum, whereas a decrease in the expression of the kinetoplastid membrane protein (KMP-11) correlated with the drug resistance phenotype [58].

Apart from the discovery of new drugs prompted by the identification of new targets, promising new fields of antiparasitic research are the approaches aimed to bring the drug directly and efficiently to the target pathogen. Drug chemistry alone is often not sufficient to fulfil this task, especially when the parasite localizes within organs, tissues or cells that behave as chemical or physical barriers to drug diffusion. Low efficiency of drug targeting requires higher dosages, and is considered one of the main causes of the serious side effects observed with most currently used antiparasitic drugs [59].

Novel formulation approaches involve the use of microparticles, nanoparticles, liposomes, niosomes or microemulsions. The polyene antibiotic amphotericin B (AmB), isolated in 1955 from Streptomyces nodosus, has a notable effect against Leishmania, but due to its toxicity it is used as a second-line treatment for emerging strains resistant to firstline products [60].

In the last decade AmB has been considered as a model for drug targeting and has been tried in many drug-carrier systems. Toxicity reduction has been achieved by the use of vehicles as lipid emulsions and unilamellar liposomes, some of which are presently in use [61]. However, the need of intravenous administration, stability and storage problems, and especially the high cost (that for some formulations is estimated to surpass one thousand dollars per patient per day) preclude their widespread use in developing countries [60]. Nanosized carriers are receiving special attention with the aim to improve drug bioavailability and selectivity, and some of them have already proved their effectiveness in the treatment and prophylaxis of malaria in animal models [62]. Basu and Lala prepared niosomes, non-ionic surfactant vesicles with a retention capacity higher than that of liposomes, that resulted suitable alternatives as drug carriers in in vitro 
and in vivo tests [63]. The same and other Authors also tested different delivery systems for an unsaturated triterpene acid isolated from Mimusops elangii [64]. The nanoparticulate form has reduced hepatotoxicity and nephrotoxicity and may be considered useful for clinical applications in human leishmaniasis. Similarly, an indolizine compound linked to phospholipid microspheres was found active in experimental leishmaniasis [65], and the nanocapsulated quercetin resulted less toxic and more potent in reducing the Leishmania burden in a hamster infection model [66]. Albumin microcapsules, that have been already validated as octafluoropropane carriers to enhance contrast in ultrasound imaging, represent an alternative that, as well as liposomes, selectively target macrophages. The encapsulation of $\mathrm{AmB}$ in albumin microparticles is cheap, and reduced toxicity improving AmB efficacy in vitro [60] and in a hamster model of $L$. infantum infection [67].

Recently, new inorganic materials with a porous structure that can be modified to control the drug release rate have been identified. These carriers are non toxic, biocompatible, and with a higher chemical and mechanical stability than organic vectors. Silica-based ordered mesoporous materials (i.e. materials that possess pores with diameters between 2 and $50 \mathrm{~nm}$ ) offer the advantage that the channels used as reservoirs to store drugs and biomolecules can be opened and closed at will by stimuli such as heat, $\mathrm{pH}$, light, chemicals, ultrasounds or magnetism [68]. So far, mesoporous materials have been tested as controlled delivery systems of antibiotics (amoxicillin, gentamicin and erythromycin) [68], and of antifungal drugs (ketoconazole, griseofulvin and itraconazole) [69]. With in vivo experiments on rabbits and dogs, Maellaerts et al. demonstrated that the systemic availability of itraconazole was significantly boosted when the drug was loaded into SBA-15, a mesoporous silicate that enhances the dissolution of drugs poorly soluble in water [70].

\section{CONCLUSIONS}

Modern drug discovery relies on different, highly sophisticated and complementary strategies that are very costly. The interaction between academic laboratories and lead discovery departments of private companies [71], coupled with new funding sources and non-for-profit partnership, is crucial to identify new active compounds [72]. Computerassisted drug design based on large molecular databases of compounds and targets are making in silico approaches like virtual screening by molecular docking more and more efficient and cost-effective [73]. In vitro experiments based on the modelling results are already underway [74].

The identification of new targets and active compounds is rapidly progressing, but it is not expected to meet the therapy needs in the close future. Therefore the short-term availability of new antiprotozoan drugs more reasonably relies on the development of already existing drugs, as is the case of ferroquine, an organometallic molecule that can overcome $P$. falciparum resistance to chloroquine and has entered phase IIb clinical trials in 2007 [75], or on the optimization of already in use effective drugs, as could be the case of antimonials in leishmaniasis. In this field, new perspectives are offered by the proven activity of orally administered meglu- mine antimoniate-b-cyclodestrin conjugates [76], and by the development of a new liposome formulation of meglumine antimoniate, that in a dog infection model was effective at a 20-fold-lower cumulative dose of $\mathrm{Sb}$ than is used for conventional antimonial treatment [77]. A third possibility is the cooptation of drugs already developed for other pathologies, as has been the case of miltefosine, an anticancer agent originally used for the local treatment of skin metastases and now also marketed for its high activity against $L$. donovani. Similarly, a new molecule now in phase III anticancer clinical trial, tipifarnib, is also currently being developed to be used against $T$. cruzi [78].

New developments are also involving the design of prelicensure phase III antimalarial trials: from tests aimed to assess the "superiority" of investigational products against the best in-use drugs, it has recently been recommended to shift to "non-inferiority" study design, in which the new therapy is deemed acceptable if it performs "at least as well as" a currently approved therapy [79]. This change should allow the licensing of a greater number of new antimalarials, that could minimize the global impact of resistance to artemisin combination therapy, should it arise.

\section{REFERENCES}

[1] Pink, R.; Hudson, A.; Mouries, A.M.; Bendig, M. Opportunities and challenges in antiparasitic drug discovery. Nat. Rev. Drug Discov., 2005, 4, 727-40.

[2] Reguera, M.R.; Diaz-Gonzalez, R.; Perez-Pertejo, Y.; BalanaFouce, R. Characterizing the bi-subunit type IB DNA topoisomerase of Leishmania parasites; a novel scenario for drug intervention in trypanosomatids. Curr. Drug Targets, 2008, 9, 966-78.

[3] Dube, A.; Gupta, R.; Singh, N. Reporter genes facilitating discovery of drugs targeting protozoan parasites. Trends Parasitol., 2009, 25, 432-9.

[4] McKerrow, H.J.; Doyle, S.P.; Engel, C.J.; Podust, M.L.; Robertson, A.S.; Ferreira, R.; Saxton, T.; Arkin, M.; Kerr, D.I.; Brinen, S.L.; Craik, S.C. Two approaches to discovering and developing new drugs for Chagas disease. Mem. Inst. Oswaldo Cruz, 2009, 104, 263-9.

[5] Kasam, V.; Zimmermann, M.; Maass, A.; Schwichtenberg, H.; Wolf, A.; Jacq, N.; Breton, V.; Hofmann-Apitius, M. Design of new plasmepsin inhibitors: a virtual high throughput screening approach on the EGEE grid. J. Chem. Inf. Model, 2007, 47, 1818-28.

[6] Jana, S.; Paliwal, J. Novel molecular targets for antimalarial chemotherapy. Int. J. Antimicrob. Agents, 2007, 30, 4-10.

[7] Loser, R.; Gutschow, M. Dipeptide-derived nitriles containing additional electrophilic sites: potentially irreversible inhibitors of cysteine proteases. J. Enzyme Inhib. Med. Chem., 2009, 24, 124552.

[8] Rosenthal, P.J.; Sijwali, P.S.; Singh, A.; Shenai, B.R. Cysteine proteases of malaria parasites: targets for chemotherapy. Curr. Pharm. Des., 2002, 8, 1659-72.

[9] Fah, C.; Hardegger, A.L.; Baitsch, L.; Schweizer, B.W.; Meyer, S.; Bur, D.; Diederich, F. New organofluorine building blocks: inhibition of the malarial aspartic proteases plasmepsin II and IV by alicyclic alpha,alpha-difluoroketone hydrates. Org. Biomol. Chem., 2009, 7, 3947-57.

[10] Hof, F.; Schutz, A.; Fah, C.; Meyer, S.; Bur, D.; Liu, J.; Goldberg, E.D.; Diederich, F. Starving the malaria parasite: inhibitors active against the aspartic proteases plasmepsins I, II, and IV. Angew. Chem. Int. Ed. Engl., 2006, 45, 2138-41.

[11] Liu, K.; Shi, H.; Xiao, H.; Chong, G.A.; Bi, X.; Chang, T.Y.; Tan, S.K.; Yada, Y.R.; Yao, Q.S. Functional profiling, identification, and inhibition of plasmepsins in intraerythrocytic malaria parasites. Angew. Chem. Int. Ed. Engl., 2009, 48, 8293-7.

[12] Friedman, R.; Caflisch, A. Discovery of plasmepsin inhibitors by fragment-based docking and consensus scoring. ChemMedChem, 2009, 4, 1317-26. 
[13] Luksch, T.; Blum, A.; Klee, N.; Diederich, E.W.; Sotriffer, A.C.; Klebe, G. Pyrrolidine derivatives as plasmepsin inhibitors: binding mode analysis assisted by molecular dynamics simulations of a highly flexible protein. ChemMedChem, 2010, 5, 443-54.

[14] Duschak, G.V.; Couto, S.A. Cruzipain, the major cysteine protease of Trypanosoma cruzi: a sulfated glycoprotein antigen as relevant candidate for vaccine development and drug target. A review. Curr. Med. Chem., 2009, 16, 3174-202.

[15] Vicik, R.; Hoerr, V.; Glaser, M.; Schultheis, M.; Hansell, E.; McKerrow, H.J.; Holzgrabe, U.; Caffrey, R.C.; Ponte-Sucre, A.; Moll, H.; Stich, A.; Schirmeister, T. Aziridine-2,3-dicarboxylate inhibitors targeting the major cysteine protease of Trypanosoma brucei as lead trypanocidal agents. Bioorg. Med. Chem. Lett., 2006, 16, 2753-7.

[16] Buback, V.; Mladenovic, M.; Engels, B.; Schirmeister, T. Rational design of improved aziridine-based inhibitors of cysteine proteases. J. Phys. Chem. B., 2009, 113, 5282-9.

[17] Ponte-Sucre, A.; Vicik, R.; Schultheis, M.; Schirmeister, T.; Moll, H. Aziridine-2,3-dicarboxylates, peptidomimetic cysteine protease inhibitors with antileishmanial activity. Antimicrob. Agents. Chemother., 2006, 50, 2439-47.

[18] Ganguly, A.; Das, B.B.; Sen, N.; Roy, A.; Dasgupta, B.S.; Majumder, K.H. 'LeishMan' topoisomerase I: an ideal chimera for unraveling the role of the small subunit of unusual bi-subunit topoisomerase I from Leishmania donovani. Nucleic Acids Res., 2006, $34,6286-97$

[19] Deterding, A.; Dungey, A.F.; Thompson, A.K.; Steverding, D. Anti-trypanosomal activities of DNA topoisomerase inhibitors. Acta Trop., 2005, 93, 311-6.

[20] Marquis, F.J.; Makhey, D.; LaVoie, J.E.; Olivier, M. Effects of topoisomerases inhibitors protoberberine on Leishmania donovani growth, macrophage function, and infection. J. Parasitol., 2003, $89,1048-52$.

[21] Proulx, E.M.; Desormeaux, A.; Marquis, F.J.; Olivier, M.; Bergeron, G.M. Treatment of visceral leishmaniasis with sterically stabilized liposomes containing camptothecin. Antimicrob. Agents Chemother., 2001, 45, 2623-7.

[22] Sen, N.; Das, B.B.; Ganguly, A.; Mukherjee, T.; Tripathi, G.; Bandyopadhyay, S.; Rakshit, S.; Sen, T.; Majumder, K.H. Camptothecin induced mitochondrial dysfunction leading to programmed cell death in unicellular hemoflagellate Leishmania donovani. Cell Death Differ., 2004, 11, 924-36.

[23] Sithiprom, S.; Petmitr, S.; Chavalitshewinkoon-Petmitr, P. Partial purification and characterization of Trichomonas vaginalis DNA topoisomerase II. Southeast Asian J. Trop. Med. Public Health, 2009, 40, 877-85.

[24] Hancock, R.E.; Scott, M.G. The role of antimicrobial peptides in animal defenses. Proc. Natl. Acad. Sci. USA, 2000, 97, 8856-61.

[25] Steinstraesser, L.; Kraneburg, U.M.; Hirsch, T.; Kesting, M.; Steinau, H.U.; Jacobsen, F.; Al-Benna, S. Host defense peptides as effector molecules of the innate immune response: a sledgehammer for drug resistance? Int. J. Mol. Sci., 2009, 10, 3951-70.

[26] Bommarius, B.; Kalman, D. Antimicrobial and host defense peptides for therapeutic use against multidrug-resistant pathogens: new hope on the horizon. IDrugs, 2009, 12, 376-80.

[27] Mor, A. Multifunctional host defense peptides: antiparasitic activities. FEBS J., 2009, 276, 6474-82.

[28] Hancock, R.E.; Rozek, A. Role of membranes in the activities of antimicrobial cationic peptides. FEMS Microbiol. Lett., 2002, 206, 143-9.

[29] Oren, Z.; Shai, Y. Mode of action of linear amphipathic alphahelical antimicrobial peptides. Biopolymers, 1998, 47, 451-63.

[30] Hwang, P.M.; Vogel, H.J. Structure-function relationships of antimicrobial peptides. Biochem. Cell. Biol., 1998, 76, 235-46.

[31] Rivas, L.; Luque-Ortega, R.J.; Andreu, D. Amphibian antimicrobial peptides and Protozoa: lessons from parasites. Biochim. Biophys. Acta, 2009, 1788, 1570-81.

[32] Savoia, D.; Guerrini, R.; Marzola, E.; Salvadori, S. Synthesis and antimicrobial activity of dermaseptin S1 analogues. Bioorg. Med. Chem., 2008, 16, 8205-9.

[33] Luque-Ortega, R.J.; van't Hof, W.; Veerman, C.E.; Saugar, M.J.; Rivas, L. Human antimicrobial peptide histatin 5 is a cellpenetrating peptide targeting mitochondrial ATP synthesis in Leishmania. FASEB J., 2008, 22, 1817-28.
[34] Sharma, K.R.; Reddy, P.R.; Tegge, W.; Jain, R. Discovery of TrpHis and His-Arg analogues as new structural classes of short antimicrobial peptides. J. Med. Chem., 2009, 52, 7421-31.

[35] Rodziewicz-Motowidlo, S.; Mickiewicz, B.; Greber, K.; Sikorska, E.; Szultka, L.; Kamysz, E.; Kamysz, W. Antimicrobial and conformational studies of the active and inactive analogues of the protegrin-1 peptide. FEBS J., 2010, 277, 1010-22.

[36] Saviello, R.M.; Malfi, S.; Campiglia, P.; Cavalli, A.; Grieco, P.; Novellino, E.; Carotenuto, A. New insight into the mechanism of action of the temporin antimicrobial peptides. Biochemistry, 2010, 49, 1477-85.

[37] Prudencio, M.; Lehmann, J.M. Illuminating the host - how RNAi screens shed light on host-pathogen interactions. Biotechnol. J. 2009, 4, 826-37.

[38] Tyc, J.; Faktorova, D.; Kriegova, E.; Jirku, M.; Vavrova, Z.; Maslov, A.D.; Lukes, J. Probing for primary functions of prohibitin in Trypanosoma brucei. Int. J. Parasitol., 2010, 40, 73-83.

[39] Price, P.H.; Guther, L.M.; Ferguson, A.M.; Smith, F.D. MyristoylCoA:protein N-myristoyltransferase depletion in trypanosomes causes avirulence and endocytic defects. Mol. Biochem. Parasitol. 2010, 169, 55-8

[40] Caceres, J.A.; Michels, A.P.; Hannaert, V. Genetic validation of aldolase and glyceraldehyde-3-phosphate dehydrogenase as drug targets in Trypanosoma brucei. Mol. Biochem. Parasitol., 2010, $169,50-4$.

[41] Monnerat, S.; Clucas, C.; Brown, E.; Mottram, C.J.; Hammarton, C.T. Searching for novel cell cycle regulators in Trypanosoma bruce $i$ with an RNA interference screen. BMC Res. Notes, 2009, 2 , 46.

[42] Vinet, F.A.; Fukuda, M.; Turco, J.S.; Descoteaux, A. The Leishmania donovani lipophosphoglycan excludes the vesicular protonATPase from phagosomes by impairing the recruitment of synaptotagmin V. PLoS Pathog., 2009, 5, e1000628.

[43] Blandin, A.S.; Wang-Sattler, R.; Lamacchia, M.; Gagneur, J.; Lycett, G.; Ning, Y.; Levashina, A.E.; Steinmetz, M.L. Dissecting the genetic basis of resistance to malaria parasites in Anopheles gambiae. Science, 2009, 326, 147-50.

[44] Ngo; H.; Tschudi; C.; Gull; K.; Ullu; E. Double-stranded RNA induces mRNA degradation in Trypanosoma brucei. Proc. Natl. Acad. Sci. U S A, 1998, 95, 14687-92.

[45] Wang, Z.; Morris, C.J.; Drew, E.M.; Englund, T.P. Inhibition of Trypanosoma brucei gene expression by RNA interference using an integratable vector with opposing T7 promoters. J. Biol. Chem., 2000, 275, 40174-9.

[46] Claser, C.; Curcio, M.; de Mello, M.S.; Silveira, V.E.; Monteiro, P.,H.; Rodrigues, M.M. Silencing cytokeratin 18 gene inhibits intracellular replication of Trypanosoma cruzi in HeLa cells but not binding and invasion of trypanosomes. BMC Cell Biol., 2008, 9 , 68 .

[47] Aouadi, M.; Tesz, J.G.; Nicoloro, M.S.; Wang, M.; Chouinard, M Soto, E.; Ostroff, R.G.; Czech, P.M. Orally delivered siRNA targeting macrophage Map4k4 suppresses systemic inflammation. $\mathrm{Na}$ ture, 2009, 458, 1180-4.

[48] Crombez, L.; Aldrian-Herrada, G.; Konate, K.; Nguyen, N.Q.; McMaster, K.G.; Brasseur, R.; Heitz, F.; Divita, G. A new potent secondary amphipathic cell-penetrating peptide for siRNA delivery into mammalian cells. Mol. Ther., 2009, 17, 95-103.

[49] Frankel, D.A.; Pabo, O.C., Cellular uptake of the tat protein from human immunodeficiency virus. Cell, 1988, 55, 1189-93.

[50] Green, M.; Loewenstein, M.P. Autonomous functional domains of chemically synthesized human immunodeficiency virus tat transactivator protein. Cell, 1988, 55, 1179-88.

[51] Endoh, T.; Ohtsuki, T. Cellular siRNA delivery using cellpenetrating peptides modified for endosomal escape. Adv. Drug. Deliv. Rev., 2009, 61, 704-9.

[52] Heitz, F.; Morris, C.M.; Divita, G. Twenty years of cell-penetrating peptides: from molecular mechanisms to therapeutics. Br. J. Pharmacol., 2009, 157, 195-206.

[53] Nekhotiaeva, N.; Elmquist, A.; Rajarao, K.G.; Hallbrink, M.; Langel, U.; Good, L. Cell entry and antimicrobial properties of eukaryotic cell-penetrating peptides. FASEB J., 2004, 18, 394-6.

[54] Arrighi, B.R.; Ebikeme, C.; Jiang, Y.; Ranford-Cartwright, L.; Barrett, P.M.; Langel, U.; Faye, I. Cell-penetrating peptide TP10 shows broad-spectrum activity against both Plasmodium falcipa- 
rum and Trypanosoma brucei brucei. Antimicrob. Agents Chemother., 2008, 52, 3414-7.

[55] Prieto, H.J.; Koncarevic, S.; Park, K.S.; Yates, J.3rd; Becker, K. Large-scale differential proteome analysis in Plasmodium falciparum under drug treatment. PLoS One, 2008, 3, e4098.

[56] Pays, E.; Vanhollebeke, B. Mutual self-defence: the trypanolytic factor story. Microbes Infect., 2008, 10, 985-9.

[57] Baral, T.N.; Magez, S.; Stijlemans, B.; Conrath, K.; Vanhollebeke, B.; Pays, E.; Muyldermans, S.; De Baetselier, P. Experimental therapy of African trypanosomiasis with a nanobody-conjugated human trypanolytic factor. Med. Sci., 2006, 12, 580-4.

[58] El Fadili, K.; Drummelsmith, J.; Roy, G.; Jardim, A.; Ouellette, M. Down regulation of KMP-11 in Leishmania infantum axenic antimony resistant amastigotes as revealed by a proteomic screen. Exp. Parasitol., 2009, 123, 51-7.

[59] Kayser, O.; Kiderlen, F.A., Delivery strategies for antiparasitics. Expert Opin. Investig. Drugs, 2003, 12, 197-207.

[60] Ordonez-Gutierrez, L.; Espada-Fernandez, R.; Dea-Ayuela, A.M.; Torrado, J.J.; Bolas-Fernandez, F.; Alunda, M.J. In vitro effect of new formulations of amphotericin B on amastigote and promastigote forms of Leishmania infantum. Int. J. Antimicrob. Agents, 2007, 30, 325-9.

[61] Torrado, J.J.; Espada, R.; Ballesteros, P.M.; Torrado-Santiago, S. Amphotericin B formulations and drug targeting. J. Pharm. Sci., 2008, 97, 2405-25.

[62] Santos-Magalhaes, S.N.; Mosqueira, C.V. Nanotechnology applied to the treatment of malaria. Adv. Drug. Deliv. Rev., 2010, 62, 560575 .

[63] Basu, K.M.; Lala, S. Macrophage specific drug delivery in experimental leishmaniasis. Curr. Mol. Med., 2004, 4, 681-9.

[64] Lala, S.; Gupta, S.; Sahu, N.P.; Mandal, D.; Mondal, N.B.; Moulik, S.P.; Basu, M.K. Critical evaluation of the therapeutic potential of basic acid incorporated in oil-in-water microemulsions and polyD,L-lactide nanoparticles against experimental leishmaniasis. $J$. Drug Target., 2006, 14, 171-9.

[65] Medda, S.; Jaisankar, P.; Manna, R.K.; Pal, B.; Giri, V.S.; Basu, M.K. Phospholipid microspheres: a novel delivery mode for targeting antileishmanial agent in experimental leishmaniasis. J. Drug Target., 2003, 11, 123-8.

[66] Sarkar, S.; Mandal, S.; Sinha, J.; Mukhopadhyay, S.; Das, N.; Basu, M.K. Quercetin: critical evaluation as an antileishmanial agent in vivo in hamsters using different vesicular delivery modes. J. Drug Target., 2002, 10, 573-8.

[67] Sanchez-Brunete, A.J.; Dea, A.M.; Rama, S.; Bolas, F.; Alunda, M.J.; Torrado-Santiago, S.; Torrado, J.J. Influence of the vehicle on the properties and efficacy of microparticles containing amphotericin B. J. Drug Target., 2005, 13, 225-33.
[68] Manzano, M.; Colilla, M.; Vallet-Regi, M. Drug delivery from ordered mesoporous matrices. Expert Opin. Drug Deliv., 2009, 6, $1383-400$.

[69] Van Speybroeck, M.; Barillaro, V.; Thi, D.T.; Mellaerts, R.; Martens, J.; Van Humbeeck, J.; Vermant, J.; Annaert, P.; Van den Mooter, G.; Augustijns, P. Ordered mesoporous silica material SBA-15: a broad-spectrum formulation platform for poorly soluble drugs. J. Pharm. Sci., 2009, 98, 2648-58.

[70] Mellaerts, R.; Mols, R.; Jammaer, A.J.; Aerts, A.C.; Annaert, P.; Van Humbeeck, J.; Van den Mooter, G.; Augustijns, P.; Martens, A.J. Increasing the oral bioavailability of the poorly water soluble drug itraconazole with ordered mesoporous silica. Eur. J. Pharm. Biopharm., 2008, 69, 223-30.

[71] Leroy, D.; Doerig, C. Drugging the Plasmodium kinome: the benefits of academia-industry synergy. Trends Pharmacol. Sci., 2008, 29, 241-9.

[72] Croft, L.S. Kinetoplastida: new therapeutic strategies. Parasite, 2008, $15,522-7$.

[73] Wolf, A.; Shahid, M.; Kasam, V.; Ziegler, W.; Hofmann-Apitius, M. In Silico Drug Discovery Approaches on Grid Computing Infrastructures. Curr. Clin. Pharmacol., 2010, 5, 37-46

[74] Kasam, V.; Salzemann, J.; Botha, M.; Dacosta, A.; Degliesposti, G.; Isea, R.; Kim, D.; Maass, A.; Kenyon, C.; Rastelli, G.; Hofmann-Apitius, M.; Breton, V. WISDOM-II: screening against multiple targets implicated in malaria using computational grid infrastructures. Malar. J., 2009, 8,88 .

[75] Chavain, N.; Davioud-Charvet, E.; Trivelli, X.; Mbeki, L.; Rottmann, M.; Brun, R.; Biot, C. Antimalarial activities of ferroquine conjugates with either glutathione reductase inhibitors or glutathione depletors via a hydrolyzable amide linker. Bioorg. Med. Chem., 2009, 17, 8048-59.

[76] Ribeiro, R.R.; Ferreira, A.W.; Martins, S.P.; Neto, L.R.; Rocha, G.O.; Le Moyec, L.; Demicheli, C.; Frezard, F. Prolonged absorption of antimony $(\mathrm{V})$ by the oral route from non-inclusion meglumine antimoniate-beta-cyclodextrin conjugates. Biopharm. Drug Dispos., 2010, 31, 109-119.

[77] Ribeiro, R.R.; Moura, P.E.; Pimentel, M.V.; Sampaio, M.W.; Silva, M.S.; Schettini, A.D.; Alves, F.C.; Melo, A.F.; Tafuri, L.W.; Demicheli, C.; Melo, N.M.; Frezard, F.; Michalick, S.M. Reduced tissue parasitic load and infectivity to sand flies in dogs naturally infected by Leishmania (Leishmania) chagasi following treatment with a liposome formulation of meglumine antimoniate. Antimicrob. Agents Chemother., 2008, 52, 2564-72.

[78] Kraus, M.J.; Verlinde, L.C.; Karimi, M.; Lepesheva, I.G.; Gelb, H.M.; Buckner, S.F. Rational modification of a candidate cancer drug for use against Chagas disease. J. Med. Chem., 2009, 52, 1639-47.

[79] Sutherland, J.C. Comparing highly efficacious antimalarial drugs. PLoS Med., 2008, 5, e228. 